\title{
Amitriptyline and Scopolamine in an Animal Model of Depression
}

\author{
R. J. KATZ ${ }^{1}$ AND S. HERSH \\ Mental Health Research Institute, Department of Psychiatry \\ University of Michigan Medical Center, Ann Arbor, MI 48109
}

Received 15 February 1980

\begin{abstract}
KATZ, R. J. AND S. HERSH. Amitriptyline and scopolamine in an animal model of depression. NEUROSCI. BIOBEHAV. REV. 5(2) 265-271, 1981.-Adult male Sprague-Dawley rats were subjected to acute (95 dB white noise) or chronic stress, or their combination. In comparison with unstressed controls, stressed rats were more active upon several measures of open field activity. A history of chronic stress eliminated the acute stress induced activation. Concurrent treatment of chronically stressed rats with amitriptyline or scopolamine, or with a combination of both drugs resulted in selective behavioral improvement (i.e., in motor activity, latency, defecation) for amitriptyline and combined treatment rats, with significant restoration of the normal behavioral response. Scopolamine however was only marginally effective. A higher dose of scopolamine proved effective, but only with a marked disruption of baseline activity. Examination of plasma corticosterone titers indicated that chronic stress induced an elevation of basal levels and that this was reversed by amitriptyline, scopolamine, and combined drug treatment. Thus while behavioral depression and elevated corticosteroids may covary they are not identically mediated.
\end{abstract}

\begin{tabular}{|c|c|c|c|c|}
\hline $\begin{array}{l}\text { Acetylcholine } \\
\text { Corticosterone }\end{array}$ & $\begin{array}{l}\text { Activity } \\
\text { Depression }\end{array}$ & $\begin{array}{l}\text { Amitriptyline } \\
\text { Open field }\end{array}$ & $\begin{array}{l}\text { Animal model of depression } \\
\text { Scopolamine }\end{array}$ & Anticholinergic \\
\hline
\end{tabular}

IN previous studies we have demonstrated the potential utility of a novel animal model of depression based upon acute and chronic stress. While normal rats become hyperactive to acute noise stress this activation is not seen if they are chronically stressed beforehand. Only antidepressant drugs appear capable of reversing the otherwise present reduction in acute stress elicited activity due to chronic stress. The model, along with most other models of its class (see Katz, [8] for a review) attempts to offer a simplified analog of a complex clinical syndrome involving sadness, dejection, gloom, anxiety, and psychosomatic complaints. Clearly, not all these dimensions can be adequately encompassed in the rat, however the animal model, as has been demonstrated in previous papers in this series, does capture some of the typical retardation of behavior and "loss of environmental engagement" which also are frequently seen clinically.

To date this model appears pharmacologically accurate and specific in its ability to identify drugs of established therapeutic benefit. Antidepressant drugs, particularly tricyclic antidepressants, possess modest to considerable anticholinergic properties. It is unlikely that anticholinergic properties are sufficient to account for all behavioral improvements in clinical depression or the present model since monoamine oxidase inhibitors are behaviorally effective (e.g., [9]) but not strongly anticholinergic. Furthermore, cholinergic blockade is not considered an accepted or effective therapy for depression [6].

Nonetheless, two factors compel a more direct and detailed examination of the effectiveness of anticholinergic drugs in the present model. As noted in previous reports, anticholinergic drugs have been falsely identified as antidepressants in other procedures [8] even in such behaviorally sophisticated techniques as leamed helplessness and behavioral despair $[1,3]$. These last procedures of course are conceptually and behaviorally similar in a number of ways to the present model. Thus, one critical test of the specificity of the present model, and its empirical relation to previous models is the ability to distinguish anticholinergic drugs from true antidepressants.

A second reason for testing a cholinergically active compound may be found in recent studies of the clinical psychobiology of depression. Janowsky and Davis have suggested that increased cholinergic tone in consort with other, particularly adrenergic, hypofunctions may contribute to depressive affect in much the same way acetylcholine modulates dopamine in Parkinson's syndrome [7]. The modulatory role is not necessarily exclusive, nor is it evident under all circumstances. A preexisting disturbance of affect or predisposition to illness may be necessary for a further psychobiologically significant role of the cholinergic system. It is therefore of interest to determine if cholinergic factors may modulate responses to antidepressants or any more specific aspects of the depressive response.

These and related issues were addressed using the preclinical model in the present experiments. The first experiment replicated the depression effect which occurs after a history of chronic stress, and examined the effects of amitriptyline, scopolamine, and their combination upon it. Amitriptyline is a drug of choice, particularly for agitated unipolar depression, and is in wide clinical use [11]. The drug has strong anticholinergic properties. Scopolamine is an anticholinergic drug not typically considered useful in the clini-

${ }^{1}$ Correspondence to Dr. Katz at Department of Psychology, Johns Hopkins University, Charles and 34th Street, Baltimore, MD 21218. 


\section{TABLE 1}

\begin{aligned} \hline Day & \multicolumn{1}{c}{ Treatment } \\ \hline 1 & Shock \\ 2 & Food deprivation $(40 \mathrm{hr}) \\ 3 &$ Cold swim \\ 4 & Water deprivation $(40 \mathrm{hr}) \\ 5 & \\ 6 &$ Heat stress \\ 7 & Shaker stress \\ 8 & Light/dark cycle reversal \\ 9 & Cold swim \\ 10 & Shock \\ 11 & Switch cage mates \\ 12 & Heat and switch cage mates \\ 13 & Remove food and increase housing density $(4 /$ cage $) \\ 14 & \\ 15 &$ Light/dark cycle reversal \\ 16 & Cold swim \\ 17 & \\ 18 & Shock \\ 19 & Water deprivation $(40 \mathrm{hr}) \\ 20 &$ Shaker \\ 21 & Test \end{aligned}

cal pharmacotherapy of depression [6]. The combined group was included to assess whether a more subtle modulatory role for scopolamine was present in conjunction with other treatments. In a second experiment, the dose of scopolamine was increased by a factor of five, to examine the effects of high drug dosages, and whether more extreme pharmacological manipulations were potentially antidepressant.

\section{GENERAL METHOD}

Subjects (double housed adult male Sprague Dawley rats; 70 days each, Charles River Farms), apparatus (a $1.22 \mathrm{~m}^{2}$ white Plexiglas open field with $4 \times 4$ grid on the floor), and chronic and acute stress procedures were identical in all respects to previous descriptions. The acute stress procedure involved exposure of individual rats to $95 \mathrm{~dB}$ of white noise for $1 \mathrm{hr}$ prior to a $6 \mathrm{~min}$ placement in the open field. The chronic stress regimen followed that given in Katz et al. [9] and is included in Table 1. The chronic stress regimen consisted of exposure to sixty minutes' of unpredictable shock (average 1 $\mathrm{mA}, 1-10$ sec duration; average 1 shock/minute ( 3 exposures)); 40 hours' food deprivation ( 2 times); cold swim at $4.0^{\circ} \mathrm{C}$ for 5 minutes ( 3 exposures); 40 hours' water deprivation ( 2 times); 5 minutes' exposure to heat stress at $40^{\circ} \mathrm{C}(2$ times); 30 minutes' shaker stress (2 times); reversal of day/night cycle ( 2 times). Stressors were delivered in a semirandom fashion every 1-3 days throughout the day/night cycle from 0:800 through 22:00 hr. Order of stress administration is presented in Table 1.

Behavioral testing involved either of two procedures. Control (basal) subjects were tested without further manipulations. Experimental subjects were exposed to an acute stress as described above. Control subjects were placed in the field without prestress. The following experimental and control groups served as the basis for statistical comparisons: (1) basal control conditions (vehicle injection, no history of chronic stress prior to testing); (2) Chronically stressed control conditions (vehicle injection during chronic stress procedure); (3) Amitryptyline groups (all chronically stressed); and (4) Scopolamine groups and combined drugs as described below. In Experiment 1, amitryptyline (5 $\mathrm{mg} / \mathrm{kg} /$ day), scopolamine ( $1 \mathrm{mg} / \mathrm{kg} /$ day), or their combination was administered for the three week stress period. All injections were intraperitoneal, $1 \mathrm{ml} / \mathrm{kg}$ in $0.9 \%$ sodium chloride vehicle. Drugs were discontinued $36 \mathrm{hr}$ prior to individual open field testing. At the close of the test rats were removed from the field and decapitated for collection of trunk blood for corticosterone assay by competitive protein binding assay using rat corticosterone as assay standard and determinations in duplicate [10].

Behavioral measures were; initial ( $3 \mathrm{~min}$ ) activity in the 12 outside squares of the field. All four feet were required to move from one square to a second for entry to be recorded; six minutes' activity in inside squares by similar movement criterion; latency to leave home square (in seconds, maximum score $=360 \mathrm{sec}$ ) bolus count; latency to initial defecation (in seconds, maximum score $=360 \mathrm{sec}$ ), and mean ranks across categories, with all groups rank ordered from most to least active across all categories except bolus count and latency to move, in which case inverse rank orders were used. Statistical analysis by univariate analysis of variance [4] followed previous descriptions, with post-hoc comparisons by Sheffe limits. Results routinely are presented as means and standard errors. Because rearing proved unreliable in all previous studies, it was not recorded in the present experiments.

\section{EXPERIMENT 1}

To initially investigate the comparative antidepressant potentials of amitriptyline and scopolamine and their combination in the behavioral model, low (for each drug approximating behavioral ED-50's as determined using independent procedures [2]) doses of these compounds were used in an incomplete factorial design. Control (vehicle) animals received chronic stress or no chronic treatment. Drug groups were chronically stressed. All groups were studied based upon control or acute stress procedures prior to behavioral testing. Thus a five (basal; chronic stress, vehicle; chronic stress, amitriptyline; chronic stress, scopolamine; chronic stress, both drugs) by two (basal vs. acute stress) design was employed.

\section{Method}

Materials and methods conform to those presented in the General Method and previous studies.

\section{Results}

As in previous studies, acute noise stress initially activated otherwise unstressed rats. This activation effect was reduced by a history of antecedent stress, however the reduction in turn was ameliorated by amitriptyline treatment but not by scopolamine which had a weak and less consistent antidepressant effect. These results are presented in detail below, and in Figs. 1 through 7.

Locomotor activity (outside squares; Fig. 1) differed reliably across groups, $F(9,67)=6.4, p<0.0001$. By post-hoc comparisons (in all cases significance is based on a critical 


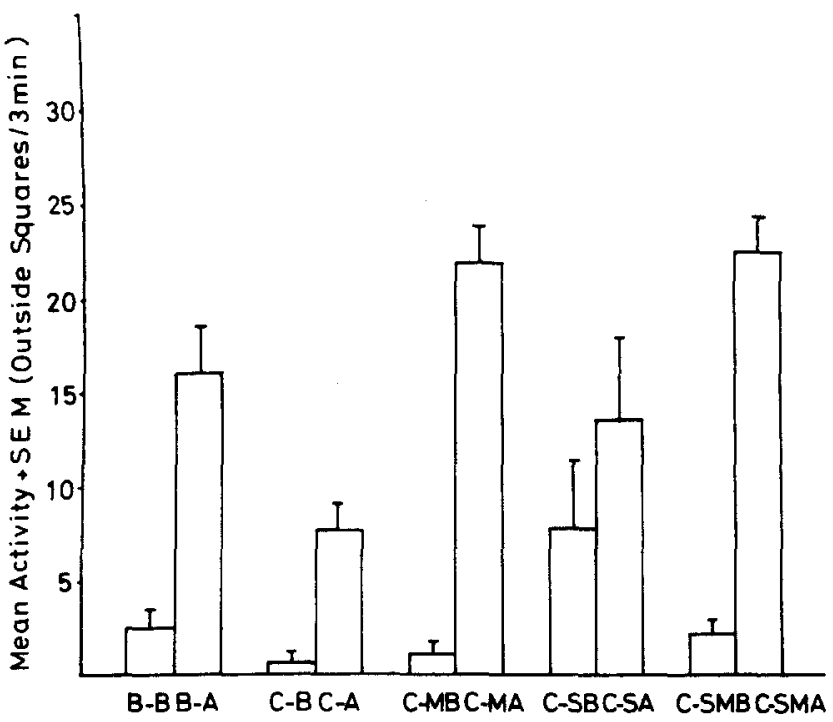

FIG. 1. Mean activity score plus standard error in an open field for rats receiving either no (basal $=B$ ) stress, acute $(=A)$ stress of $95 \mathrm{~dB}$ white noise, or a history of 3 weeks chronic $(=C)$ stress along with the tricyclic antidepressant amitriptyline (M) $5 \mathrm{mg} / \mathrm{kg} /$ day, the anticholinergic drug scopolamine $1 \mathrm{mg} / \mathrm{kg} /$ day $(S)$ or their combinaton (SM). The leftmost two columns are the non-chronically stressed control, i.e., rats receiving no stress (left) or acute stress (right); 2nd set of columns from left is chronically stressed, 3rd set of columns from left is chronically stressed with amitriptyline, 4th set of columns is chronically stressed with scopolamine, final set of columns is chronically stressed with both drugs. The convention of no acute stress; acute stress in left, right order is used throughout. Initial three minutes' grid crossings are recorded during a single placement in the field.

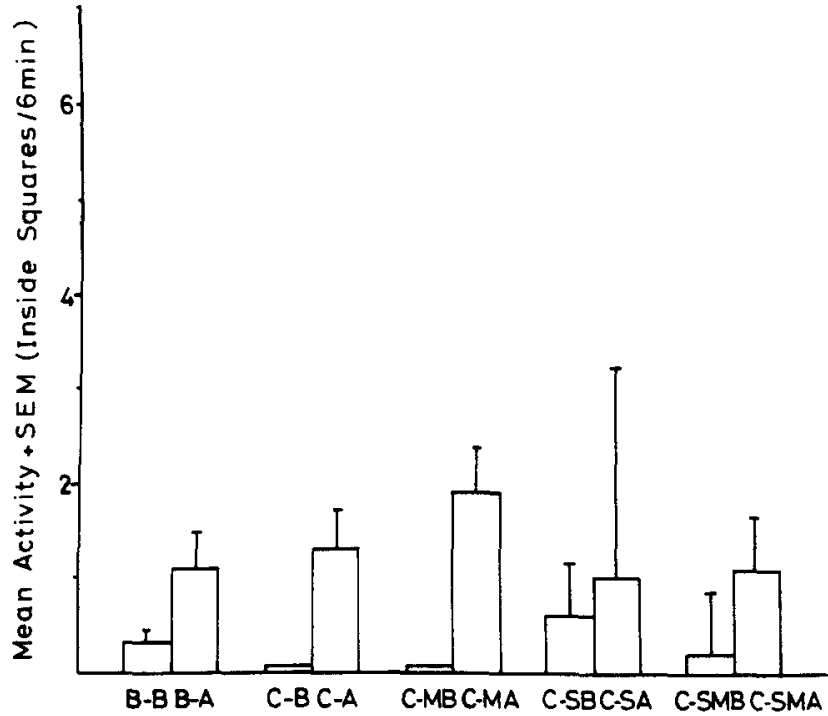

FIG. 2. Center field penetration in an open field. Mean score plus standard error for a six minute placement are shown. Rats received acute or chronic stress with three drug treatments in conjunction with the latter. $B=$ basal (not stressed), $A=$ acutely stressed for $1 \mathrm{hr}$, $\mathrm{M}=$ chronically treated with amitriptyline, $\mathrm{S}=$ chronically treated with scopolamine. The leftmost two columns are the non-chronically stressed control i.e. rats receiving no stress (left) or acute stress (right); 2nd set of columns from left is chronically stressed, 3rd set of columns from left is chronically stressed with amitriptyline, 4th set of columns is chronically stressed with scopolamine, final set of columns is chronically stressed with both drugs. The convention of no acute stress; acute stress in left, right order is used throughout. difference smaller than $p<0.05)$ indicated this was due to acute $(60 \mathrm{~min})$ stress-induced increases in activity specific to otherwise untreated (i.e., vehicle injected) rats $(F=8.0)$. The activation affect also was seen in amitriptyline treated chronically stressed rats $(F=15.1)$ and in chronically treated rats receiving combined treatment $(F=15.9)$. Chronically stressed saline or scopolamine treated rats did not show an acute activation $(F=2.0,2.5 ;$ n.s. $)$.

Inside squares (Fig. 2) did not show a significant main effect, $\mathrm{F}(9,67)=2.4, p=0.2$, precluding further analysis.

Initial Movement Latency Scores (Fig. 3) showed reliable group differences, $F(9,67)=4.3, p<0.0002$. Latency to movement was significantly reduced in vehicle treated rats by acute stress $(F=11.2)$. Acute stress was marginally but not significantly effective in chronically stressed rats $(F=1.9)$. Amitriptyline and combined drug treatment restored normal responsiveness to acute stress in chronically treated rats $(F=6.9,7.7)$. This effect was not present with scopolamine $(F=2.2)$.

Defecation scores (Mean Bolus Count: Fig. 4) differed across groups, $\mathrm{F}(9,67)=3.2, p<0.005$, with significant stress induced reductions in vehicle treated rats $(F=4.4)$ and amitriptyline treated rats $(F=7.8)$. This acute stress induced reduction in normal defecation was absent after other chronic stress treatments (for vehicle, scopolamine, and combined drug F's $<1$ ).

Analysis of defecation latency (Fig. 5) indicated a significant groups effect, $F(9,67)=3.8, p<0.001$. Latencies were increased by acute stress in vehicle treated control rats $(F=4.6)$ but not in chronically stressed rats $(F ' s<1)$. Amitriptyline restored the latency increase $(F=6.9)$ while scopolamine and a combination of drugs were not effective (both F's $<1$ ).

Corticosterone data (Fig. 6) provided supplementary support for the above findings. An overall effect of groups was evident, $F(9,67)=6.1, p<0.0001$. For all groups (acute, chronic vehicle, amitriptyline, scopolamine, combined), acute stress produced a significant elevation of circulating corticosteroids ( $F=6.1,4.9,6.3,6.0,5.8$, respectively). It might also be noted that chronic stress elevated basal steroid levels in comparison with rats not receiving the three week procedure $(F=3.7)$ and this was restored to baseline by amitriptyline, scopolamine and their combined treatment $(F=3.6,3.0,2.7)$.

Finally, Fig. 7 presents an analysis of composite mean scores across categories based upon relative ranks. The major findings from this last analysis are an increased overall level of activation in otherwise basal rats $(F=14.0)$ which 


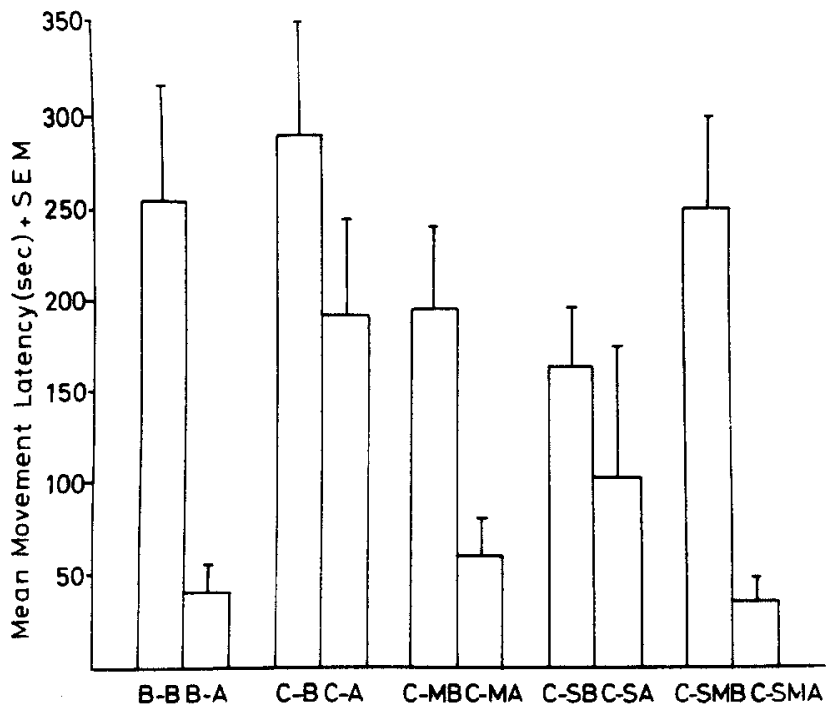

FIG. 3. Mean latencies (in sec) to initial activity in an open field plus standard errors. Rats received acute or chronic stress, with three drug treatments in conjunction with the latter. The leftmost two columns are the non-chronically stressed control, i.e., rats receiving no stress (left) or acute stress (right); 2nd set of columns from left is chronically stressed, 3rd set of columns from left is chronically stressed with amitriptyline, 4th set of columns is chronically stressed with scopolamine, final set of columns is chronically stressed with both drugs. The convention of no acute stress; acute stress in left, right order is used throughout. Complete figure legend is presented in Fig. 1.

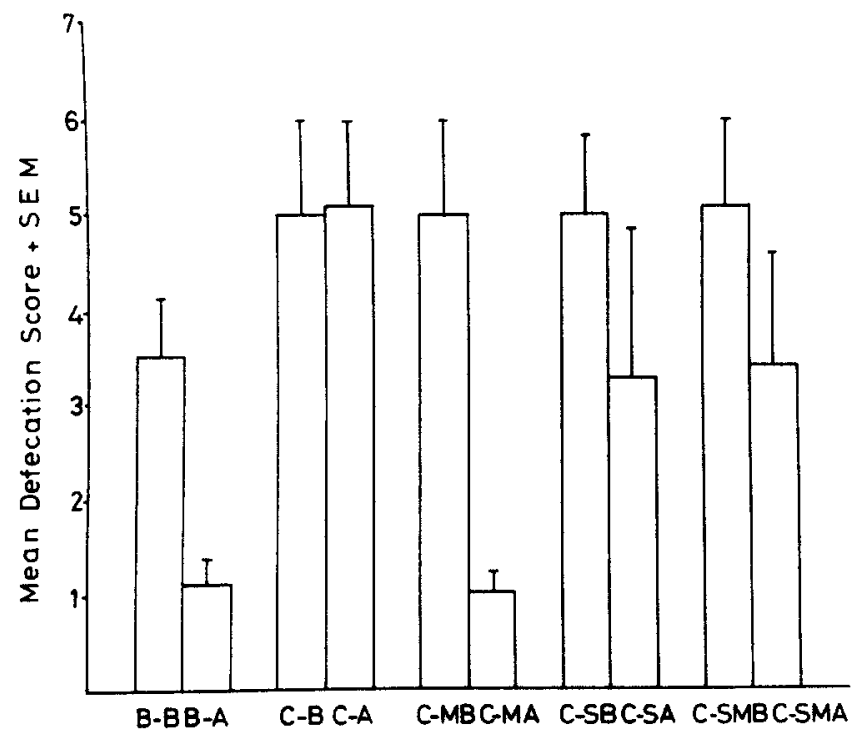

FIG. 4. Mean defecation score in an open field plus standard error. Bolus count is presented. Rats received either acute or chronic stress, with three drug treatments in conjunction with the latter. The leftmost two columns are the non-chronically stressed control, i.e., rats receiving no stress (left) or acute stress (right); 2 nd set of columns from left is chronically stressed, 3rd set of columns from left is chronically stressed with amitriptyline, 4 th set of columns is chronically stressed with scopolamine, final set of columns is chronically stressed with both drugs. The convention of no acute stress; acute stress in left, right order is used throughout. Complete figure legend is presented in Fig. 1.

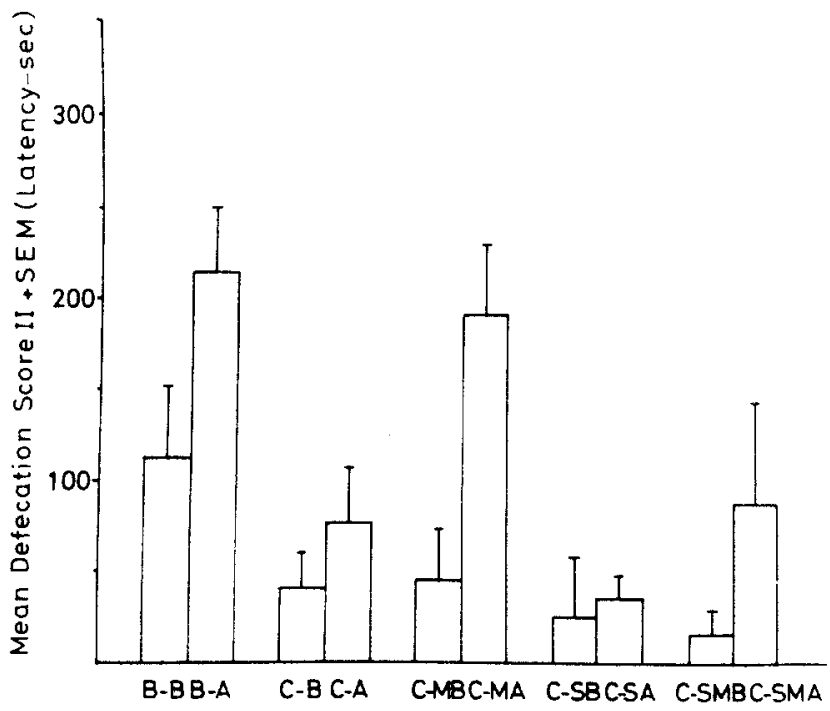

FIG. 5. Mean defecation latencies (in sec) in an open field plus standard errors. Rats received either acute or chronic stress, with three drug treatments in conjunction with the latter. The leftmost two columns are the non-chronically stressed control, i.e., rats receiving no stress (left) or acute stress (right); 2nd set of columns from left is chronically stressed. 3rd set of columns from left is chronically stressed with amitriptyline, 4th set of columns is chronically stressed with scopolamine, final set of columns is chronically stressed with both drugs. The convention of no acute stress; acute stress in left, right order is used throughout. Complete figure legend is presented in Fig. 1.

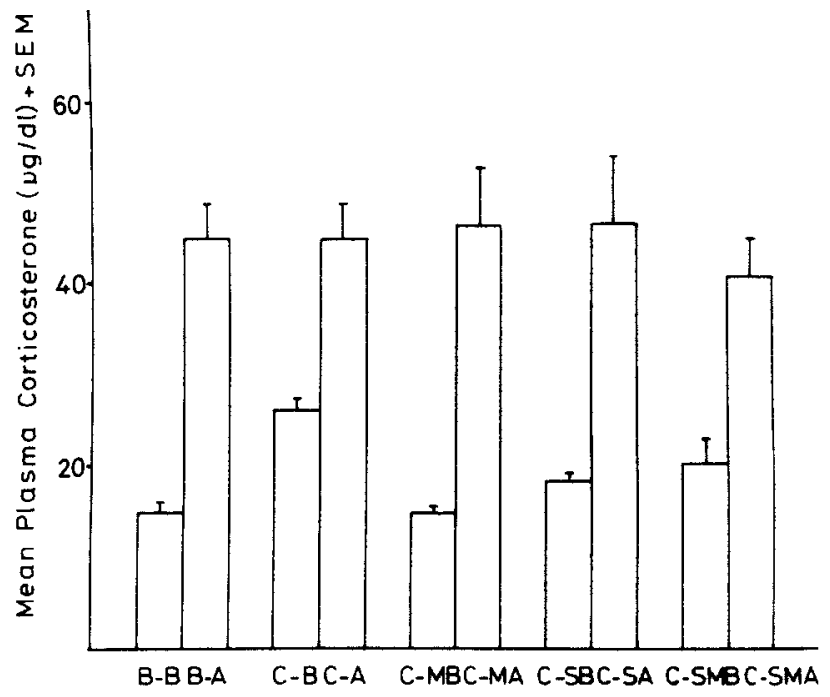

FIG. 6. Mean plasma corticosterone levels (in $\mu \mathrm{g} / \mathrm{dL}$ ) plus standard errors. All rats were exposed to acute or chronic stress, with three drug treatments in conjunction with the latter. A six minute open field test was followed by immediate removal and decapitation. Trunk blood was collected and used in competitive protein binding assay as described in text. Complete figure legend is presented in Fig. 1. The leftmost two columns are the non-chronically stressed control, i.e., rats receiving no stress (left) or acute stress (right); 2 nd set of columns from left is chronically stressed, 3rd set of columns from left is chronically stressed with amitriptyline, 4th set of columns is chronically stressed with scopolamine, final set of columns is chronically stressed with both drugs. The convention of no acute stress; acute stress in left, right order is used throughout. 


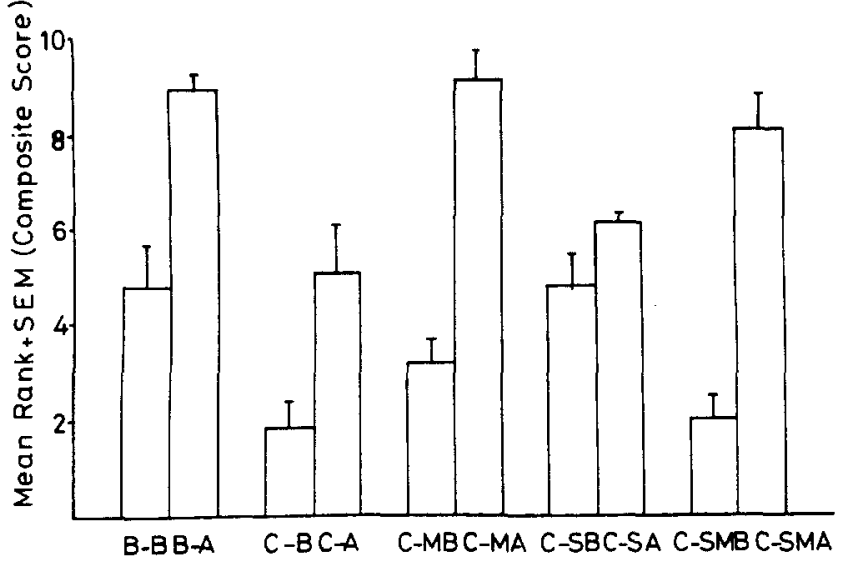

FIG. 7. Composite activity score for open field activity of rats in Experiment 1 . The leftmost two columns are the non-chronically stressed control, i.e., rats receiving no stress (left) or acute stress (right); 2nd set of columns from left is chronically stressed, 3rd set of columns from left is chronically stressed with amitriptyline, 4th set of columns is chronically stressed with scopolamine, final set of columns is chronically stressed with both drugs. The convention of no acute stress; acute stress in left, right order is used throughout. Scores calculated as mean relative ranks across categories.

was significantly reduced by chronic stress $(F=3.1)$, and chronic stress with scopolamine treatment $(F=1.7)$. Both amitriptyline $(F=10.1)$ and combined drug treatment $(F=25.0)$ were effective in restoring the acute stress response in chronically stressed rats.

\section{Discussion}

In the first experiment amitriptyline significantly improved otherwise depressed open field activation, and reduced a concomitant elevation of basal corticosterone levels. The anticholinergic drug was less effective in altering behavior although it too restored pituitary-adrenal activity towards normal. Combined drug treatment more closely resembled amitriptyline, but was slightly less effective.

To test the influence of anticholinergic factors in the restoration of function, drug doses were initially equated as behavioral ED-50's. If anticholinergic factors were sufficient for an antidepressant response, both treatments should have been equally effective. They clearly were not. It follows other factors must be involved in the reversal effect. Examination of corticosterone data indicated that both treatments were roughly equivalent at an endocrine level. Cholinergic neurons therefore may normally enhance pituitary-adrenal responsiveness in chronic stress and be sufficient for the reported endocrine effects but not behavioral improvements. This finding suggests a more limited role for cholinergic factors and emphasizes that, at least upon certain criteria, the drugs indeed were roughly equivalent. These last results on pituitary-adrenal control in rats are also consistent with studies on the normal regulation of pituitary adrenal activity in humans [5].

These findings stand in contrast to other reports using related behavioral approaches. Cholinergic antagonists have been reported effective in the learned helplessness model [1] and in behavioral despair [3]. It should be emphasized that the dosages reported effective in both these last studies are close to or identical with the dose used in the present study. Since different species, stress histories, courses of drug administration and testing procedures were employed it should not be surprising that the present and previous models also differ pharmacologically.

\section{EXPERIMENT 2}

Experiment 1 suggested that while amitriptyline was an effective treatment in the present model, scopolamine was not. Cholinergic blockade brought about modest but unreliable behavioral improvements. The dose of scopolamine was effective as judged both from other related treatments (e.g., $[1,2,3]$ and the above endocrine data). It is possible that a higher degree of anticholinergic activity is necessary for behavioral effects. The second experiment increased the dose of scopolamine fivefold, and examined effects using a factorial design.

\section{Method}

Subjects, apparatus and behavioral procedure were identical to previous description. A complete $2 \times 2 \times 2$ factorial design (presence vs. absence of acute stress, presence vs. absence of chronic stress, vehicle vs. drug) was employed using a higher dose $(5 \mathrm{mg} / \mathrm{kg} /$ day) of scopolamine. Corticosterone was not analyzed at the close of the experiment.

\section{Results}

The findings confirm the existence of stress elicited arousal and its reduction by chronic stress. Very high doses of scopolamine eliminated the otherwise present depression of open field activity and alterations in defecation measures, but only with a concomitant nonspecific disruption of baseline activity. The findings are presented below and in Figs. 8-11.

Locomotor activity (Fig. 8; outside squares/three minutes) was reliably different across cells, $F(7,40)=5.5$, $p<0.001$, with significant stress induced elevations in otherwise basal rats $(F=5.6)$ which were eliminated by chronic stress ( $F$ 's $<1)$. All scopolamine groups were elevated from a nonstress nondrugged baseline (F's respectively $=6.0,11.6,8.6,13.4$ for basal and stressed groups with and without chronic stress). None of the last groups differed among themselves (in all cases F's $<1$ ). Thus, while vehicle treated rats showed a typical pattern of activity and activity reduction, high doses of drug produced an elevation in activity unrelated to acute or chronic stress.

Inside squares were not significantly altered by the treatment conditions, $F(7,40)=0.9$, precluding further analysis. In the interest of brevity, the data are not presented in greater detail.

Initial motor latency (Fig. 9) was reliably different across cells, $\mathrm{F}(7,40)=4.8, p<0.001$. Activity was reduced in otherwise basal rats by acute stress $(F=7.9)$. It was significantly reduced in chronically stressed rats as well $(F=9.8)$ but to a reliably smaller degree $(F=3.9)$. In all cases but chronic stress without acute stress, scopolamine groups were reliably different from basally unstressed groups $(F=6.9,9.2$, $0.1,5.2$ ). Since scopolamine had its most pronounced effect upon unstressed rats and there are no reliable differences $(F ' s<1)$ in the responses of drug treated animals with respect to a prior history of chronic stress, this supports a nonspecific interpretation of the results. 


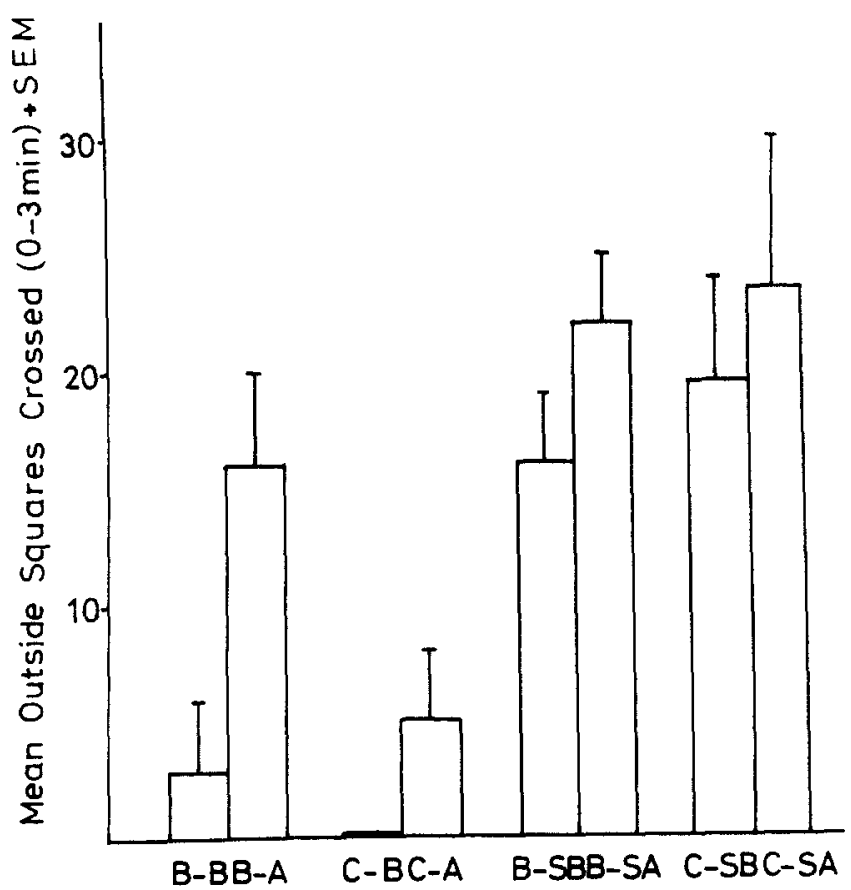

FIG. 8. Mean locomotor activity (outside squares/3 min) plus standard error in a $2 \times 2 \times 2$ factorial design (Acute stress (A) vs. Basal (B); 3 weeks' chronic stress (C) vs. Basal (B); Scopolamine (S) 5 $\mathrm{mg} / \mathrm{kg} /$ day during chronic stress vs. vehicle).

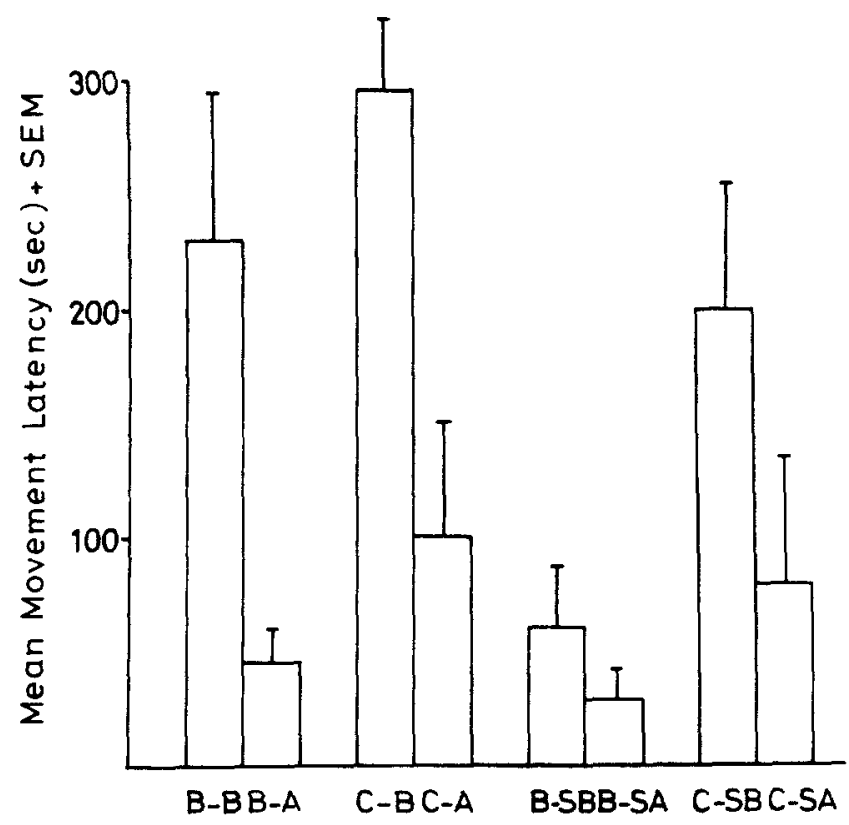

FIG. 9. Mean latencies (in sec) to initial activity plus standard error in a $2 \times 2 \times 2$ factorial design using a higher dose of scopolamine. Figure legend is further described in Figs. 1 and 8.

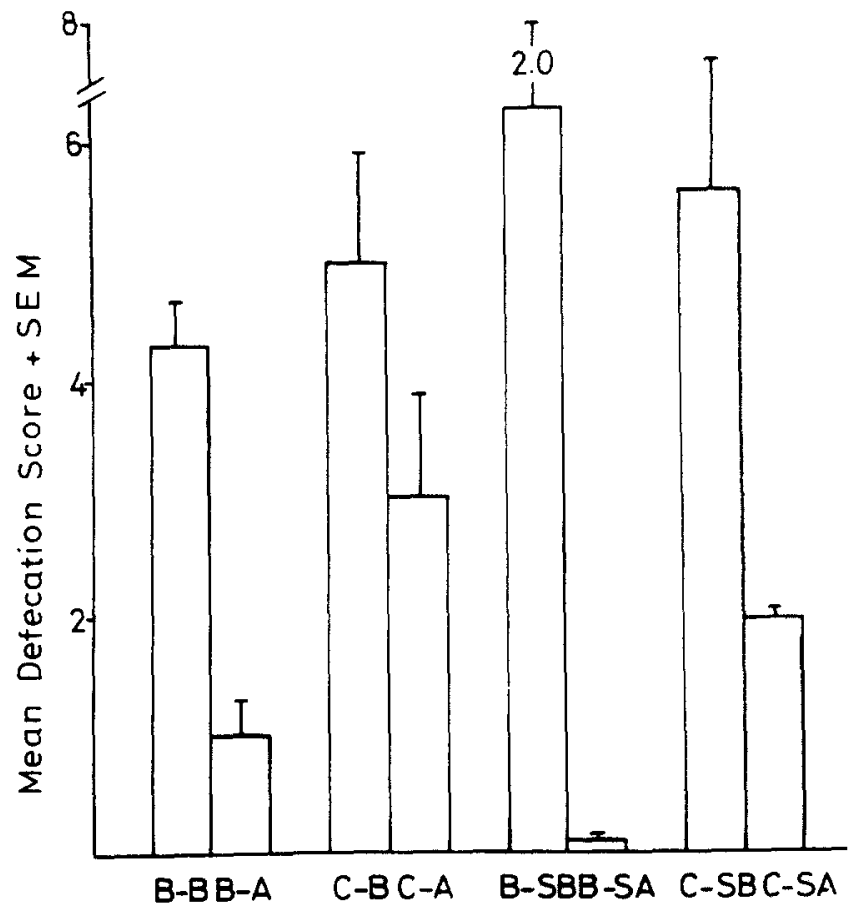

FIG. 10. Mean defecation score (bolus count) plus standard error in an open field in a $2 \times 2 \times 2$ factorial design using a higher dose of scopolamine. Figure legend is further described in Figs. 1 and 8.

Defecation scores (Fig. 10) also differed from each other in statistically reliable fashion, $F(7,40)=4.6, p<0.001$, based upon significant acute stress induced reductions of defecation in otherwise basal rats $(F=5.7)$, which in turn were significantly reduced from this level by chronic stress $(F=8.2)$, and were not themselves significant. Scopolamine was effective in restoring the normal stress induced inhibition in both basal and chronically stressed rats ( $F$ 's $=15.5,6.8$ ).

Defecation latencies (Fig. 11) followed a similar pattern ( $F$ for main effect $=3.8, p<0.005$ ). Stress effects were reliably present for both non-chronically treated acutely stressed groups in comparison to basal controls ( $F$ 's $=0.2$, 1.3 , respectively, for vehicle and scopolamine), indicating a drug effect upon baseline but no antidepressant effects for scopolamine on this last measure.

\section{Discussion}

The second experiment utilized a higher dose of scopolamine than the first experiment, but did not find a general reversal of open field depression similar to that seen with amitriptyline in the first experiment. Rather, scopolamine's effects upon behavior reflected nonspecific disinhibition. The present results suggest either that antidepressant effects were absent on individual measures, or that such effects occurred only with grossly aberrant behavioral baselines. Thus, the results cannot reasonably be attributed to effects unique to the chronic stress procedure. These findings argue for caution in ascribing preclinical antidepressant potential to anticholinergics and suggest a continuing need to examine effects from the vantage point of both normal and abnormal behavioral baselines. 


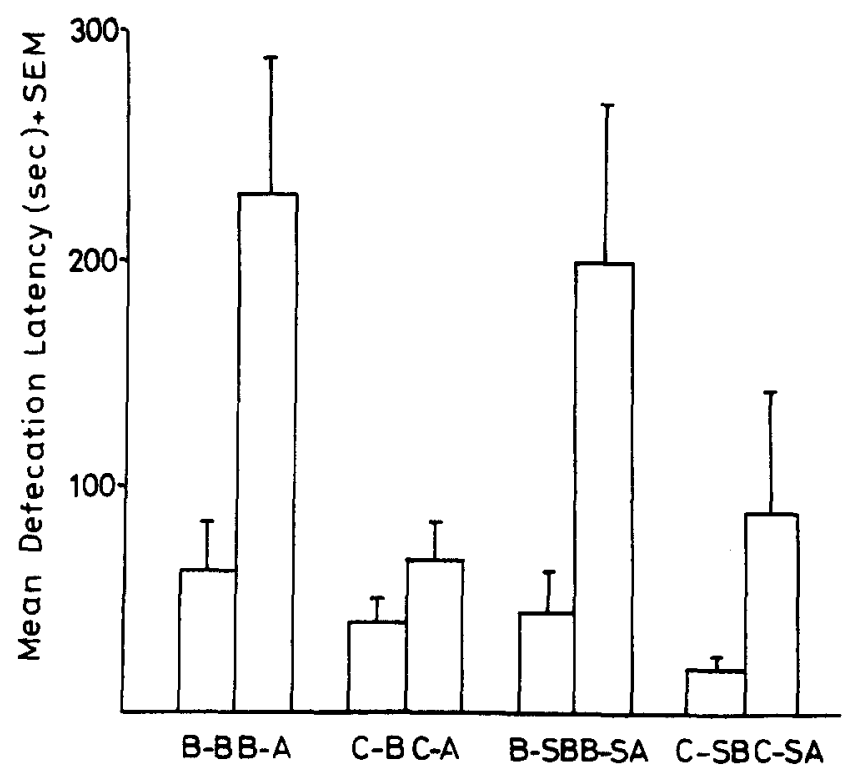

FIG. 11. Mean defecation latency (in sec) plus standard error, in an open field in a $2 \times 2 \times 2$ factorial design using a higher dose of scopolamine. Figure legend is further described in Figs. 1 and 8.

\section{GENERAL DISCUSSION}

Only limited evidence supports a direct cholinergic role in the clinical symptomatology of depression $[6,7]$. We do not deny the existence of cholinergic factors in depression, however they must be seen as one category of effect in a more complex biological disruption. Cholinergic neurons may well modulate endocrine control in the animal model of depression, and their blockade may have a mild but limited degree of behavioral benefit. Cholinergic blockade per se, however, is clearly less effective in the present model than amitriptyline, a tricyclic drug with anticholinergic properties. These results further suggest the animal model is accurate, specific, and at least to a degree distinctive from other animal models.

In closing however we reemphasize that the model is limited in its characterization of the far more complex clinical presentation of depression in humans. Insofar as rats show some behaviors analogous to human coping the present model seems to embody at least certain common factors. It is not our present intention to augue for strict isomorphic correspondence but rather for the existence of some common ground between the laboratory model and clinical findings which can be used for further investigation.

\section{ACKNOWLEDGMENTS}

The statistical assistance of Robert Shea and editorial assistance of Abe Feingold are acknowledged with thanks. Portions of this work were carried out by the second author in partial fulfillment of a graduate course requirement in psychology (No. 619) at the University of Michigan. Supported by grant MH 31588 to Drs. B. J. Carroll (primary investigator) and R. J. Katz (co-investigator). Dr. Katz is a Sloan Foundation Fellow.

\section{REFERENCES}

1. Anisman, H., G. Remington and L. S. Sklar. Effect of inescapable shock on subsequent escape performance catechola. minergic and cholinergic mediation of response initiation and maintenance. Psychopharmacology 61: 107-124, 1979.

2. Barnes, C. D. and L. G. Eltherington. Drug Dosage in Laboratory Animals. Berkeley, Calif.: University of California Press, 1964.

3. Browne, R. G. Effects of antidepressants and anticholinergics in a mouse "behavior despair" test. Eur. J Pharmac. 58: 331-334, 1979.

4. Bruning, J. L. and B. L. Klintz. Computational Handbook of Statistics, 2nd edition. Glenview, Ill.: Scott Foresman Co., 1977.

5. Carroll, B. J., J. F. Greden, R. Haskett, M. Feinberg, A. A. Albala, F. I. R. Martin, R. T. Rubin, B. Heath, P. T. Sharp, W. L. McLeod and M. F. McLeod. Neurotransmitter studies of neuroendocrine pathology in depression. Acta psych. scand. 61 (Supp 280) 183-200, 1980.
6. Innes, I. R. and M. Nickerson. Atropine, scopolamine, and related antimuscarinic drugs. In: The Pharmacological Basis of Therapeutics, edited by L. S. Goodman and A. Gilman. New York: MacMillan, 1975, pp. 514-532.

7. Janowsky, D. S. and J. M. Davis. Psychological effects of cholinominetic agents. In: Brain Acerylcholine and Neuropsychiatric Disease, edited by K. L. Davis and P. A. Berger. New York: Plenum Press, 1979, pp. 3-14.

8. Katz, R. J. Animal models and human depressive disorders. Neurosci. Biobehar. Rev. 5: 231-246, 1981.

9. Katz, R. J., K. A. Roth and K. Schmaltz. Amphetamine and tranylcypromine in an animal model of depression: Pharmacological specificity of the reversal effect. Neurosci. Biobehav. Rev. 5: 259-264, 1981.

10. Murphy, B. E. P. Some studies of the protein binding of steroids and their application to the routine micro and ultra micro measurement of various steroids in body fluids by competitive protein binding radioassay. J. clin. Endocr. 27: 973-990, 1967.

11. Van Praag, H. M. Psychotropic Drugs-A Guide for the Practitioner. New York: Brunning/Mazel Inc., 1978. 